\title{
Selection and comparison of equipment for deagglomeration processes
}

\author{
Jerzy Bałdyga, Katarzyna Malik \\ Warsaw University of Technology, Faculty of Chemical and Process Engineering, ul. Waryńskiego 1, 00-645 Warszawa, \\ Poland, e-mail: baldyga@ichip.pw.edu.pl
}

\begin{abstract}
The unique properties of nanoparticles and nanoparticle clusters show high potential for nanomaterials to be formulated into numerous products. In this paper, nanosuspensions are formulated by braking up agglomerates in high-shear flows. The flows are generated in the specific equipment, and this paper serves as a guide for equipment selection based on mechanistic modelling. A general model based on the power input to the system is formulated to model agglomerate disintegration in different types of equipment including stirred tanks, the rotor-stator disintegrators, the high-pressure nozzle systems and bead mills. The results of computations based on the rate of energy dissipation are presented in terms of the specific energy input, which is typical of industrial applications. In the considered deagglomeration devices the stresses are generated due to various mechanisms including the effects of hydrodynamic stresses, cavitation and bead collisions. The model includes the effects of agglomerate structure on the suspension viscosity. The results of the simulations are compared with the experimental data.
\end{abstract}

Keywords: Aggregates, Agglomerates, De-agglomeration, Disintegration, Suspension.

\section{INTRODUCTION}

The technological aspects of this paper are related to the formulation of stable suspension of nanoparticles. The unique properties of nanoparticles and nanoparticle clusters have shown huge potential for nanomaterials to be formulated into numerous high quality products. This includes abrasion-resistant transparent coatings, polishing slurries for optics, catalysts, sunscreen lotions, as well as the healthcare, medicine and electronics materials. In this paper, we consider the nanosuspensions that are formulated by braking up agglomerates in high-shear flows.

Disintegration processes play a significant role in chemical industry. There are many types of equipment (e.g. rotor-stators, high pressure nozzles devices, stirred tanks) that can be applied to generate stresses. There are, of course different mechanisms involved in stress generation and thus different mechanisms of deagglomeration. For example, in the case of high-pressure system disintegration results from hydrodynamic and cavitation stresses, in the case of the rotor-stator system, which is considered in this work, only hydrodynamic stresses are active. For the milling systems stresses are generated due to the milling beads collisions. To choose the most suitable equipment for the desired parameters of the final product one can use relatively simple mechanistic modelling based on the rate of energy dissipation and specific energy input calculations. The model applied in this paper includes the effects of agglomerate structure and suspension rheology on the disintegration processes.

\section{STRUCTURE AND STRENGTH OF AGGLOMERATES}

The strength of agglomerate depends on its structure, which determines the mechanism of its breakage. A typical agglomerate structure is represented by the clusterfractal model ${ }^{1}$ as shown in Fig. 1. Large clusters of size $L_{i}$ consist of smaller primary aggregates of a size $L_{a}$, which are composed of primary particles of size $L_{0}$. They are characterised by the fractal dimension $D_{f}$. The primary particles, forming primary aggregates are connected by strong chemical bonds. Such aggregates are strong, stable and resistant to mechanical forces. They are characterized by average fractal dimension $D_{f 0}$. The clusters of size $L_{i}$, connected by adhesion forces, are relatively unstable and can be disintegrated by mechanical forces.

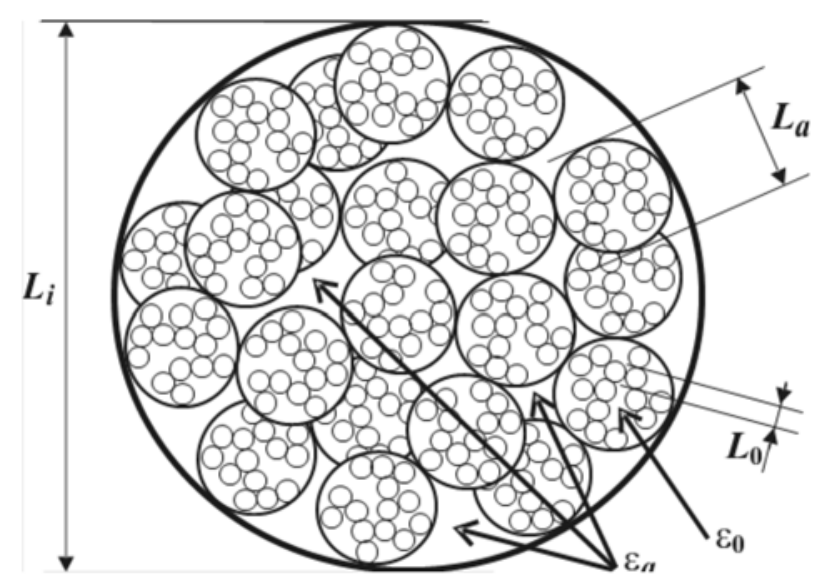

Figure 1. Structure of large agglomerates (clusters)

Alternatively to the cluster-fractal structure one can consider agglomerates of size $L_{i}$, consisting of primary particles of size $L_{0}$ that are connected by adhesion forces and characterised by the fractal dimension $D_{f}$. Then deagglomeration leads directly to primary particles of size $L_{0}$. Notice that the cluster-fractal model can be easily reduced to the single fractal model, so in what follows the cluster-fractal model is presented in detail and applied in modelling. The model of agglomerate structure applied in this work and presented in Fig.1 was published earlier by Bałdyga et al. ${ }^{2}$.

According to this model, the porosity of primary aggregates,

$\varepsilon_{0}=1-\frac{\theta_{p}^{\frac{D_{f 0}}{3}} \delta_{p}^{\frac{D_{f 0}}{3}-1}}{\delta_{p 0}^{\frac{D_{f 0}}{3}-1}}\left(\frac{L_{a}}{L_{0}}\right)^{D_{f 0}-3}$ 
and the effective volume fraction of primary aggregates in the suspension can be calculated using the fractal geometry

$\phi_{e f f, a}=\phi_{0}\left(\frac{L_{a}}{L_{0}}\right)^{3-D_{f 0}}$

where $\theta_{\mathrm{p}}$ is packing factor, $\delta_{\mathrm{p}}$ and $\delta_{\mathrm{p} 0}$ are the shape factors of aggregates and primary particles, respectively and $\phi_{0}$ is a true volume fraction of primary particles.

For the large clusters the effective porosity takes the form

$\varepsilon_{a}=1-\frac{\theta_{a}^{\frac{D_{f}}{3}} \delta_{a}^{\frac{D_{f}}{3}-1}}{\delta_{p}^{\frac{D_{f}}{3}-1}}\left(\frac{L_{i}}{L_{a}}\right)^{D_{f}-3}$

and the effective volume fraction of agglomerates reads

$\phi_{e f f}=\phi_{e f f, a}\left(\frac{L_{i}}{L_{a}}\right)^{3-D_{f}}$

The tensile strength of large agglomerates that results from adhesion (intermolecular polar and electrostatic forces) is then calculated using the model presented by Tang et al. ${ }^{3}$

$\sigma_{T}=1.1 \frac{1-\varepsilon_{a}}{\varepsilon_{a}} \frac{F}{L_{a}^{2}}$

where $F$ represents the force of interaction between primary aggregates forming an agglomerate. The forces can be calculated as the sum of the van der Waals attractive force $\left(F_{\mathrm{A}}\right)$ and the electrostatic repulsive force $\left(F_{\mathrm{R}}\right)$. Both forces can be calculated using the classical DLVO theory: $F=F_{\mathrm{A}}+F_{\mathrm{R}}$

The van der Waals and electrostatic forces act between primary particles and are expressed for $R_{0}=L_{0} / 2$ by:

$F_{A}=-\frac{H a R_{0}}{24 H^{2}}$

$F_{R}=\frac{16 \pi \chi R_{0} k_{B} T^{2}}{e^{2} z^{2} H}\left\{\frac{\exp \left[\frac{z e \psi_{d}}{\left(2 k_{B} T\right)}\right]-1}{\exp \left[\frac{z e \psi_{d}}{\left(2 k_{B} T\right)}\right]+1}\right\}^{2} \exp (-\kappa H)$

where $H a$ is an effective Hamaker constant, $H$ is the surface-to-surface distance, $\chi$ static permittivity, $k_{\mathrm{B}}$, the Boltzman constant, $\mathrm{T}$ denotes temperature, $e$ electron charge, and $\kappa$ the Debye-Hückel parameter

$\kappa=\sqrt{\frac{2 e^{2} N_{A V} I_{s}}{\chi k_{B} T}}$

In eq.(9) $N_{\mathrm{AV}}$ represents the Avogadro number and $I_{s}$ is the ionic strength.

The surface potential, $\psi_{\mathrm{d}}$, can be measured as zeta potential or can be calculated as the Nernst surface potential using the so-called Gouy-Chapman-Stern-Grahame approximation

$\Psi_{0 i}=\frac{k_{B} T}{z e} \ln \ln \left(\frac{c_{x^{z+}}}{c_{x^{z+}, i s o}}\right)-\frac{\sigma}{C_{i n}}$ where $C_{\mathrm{in}}$ is the inner layer capacitance per unit area, $c_{\mathrm{x}}^{\mathrm{z}+}$ is ion concentration in the solution, $c_{\mathrm{x}}{ }^{\mathrm{z}+}$,iso is ion concentration at the isoelectric point and $\sigma$ represents the surface charge. The charge and the surface potentials are connected by the Poisson-Boltzman approximation that is based on Boltzman expression ${ }^{4}$ that for symmetrical $z-z$ charge electrolytes takes the form

$\sigma=-2 z e n_{0} \sinh \sinh \left(\frac{z e \Psi_{0 i}}{k_{B} T}\right)$

where $n_{0}$ is the concentration of each ion in the bulk solution. For the asymmetrical electrolytes one can use

$\Psi_{0 i}=\frac{k_{B} T}{z e} \ln \ln \left(\frac{c_{x^{z+}}}{c_{x^{z+}, i s o}}\right)$

Notice that for the surface-to-surface distance, $H$, close to zero, the ratio of repulsive to attractive forces that is proportional to $H$, approaches zero, and the repulsive forces are negligible as compared to attractive ones. However, the repulsive forces can dominate particle interactions when the distance between particles is large, which can stabilize the suspension. This, however, cannot happen for the concentration of potential-controlling ions close to the isoelectric point, when the surface potential is equal to zero.

\section{PROBLEM OF SUSPENSION VISCOSITY}

The suspended particles can affect the suspension viscosity and thus the flow pattern. Hence, the viscosity model must be incorporated into the calculations.

The suspension viscosity can be expressed in the form: $\mu=\mu_{0} M$

where $\mu_{0}$ is the suspending fluid viscosity and $M$ is relative suspension viscosity, which depends on the effective volume fraction of particles. In the literature there are many models that allow the prediction of particle concentration effect on suspension viscosity. For example, Collins ${ }^{5}$ proposed to use the extended Krieger Dougherty model in the following form:

$M=\left(1-\frac{\phi_{0}}{A}\right)^{-2.5}$

where $A$ for fractal aggregates takes the form

$A=N^{\left(D_{f}-3\right) / D_{f}}-\left(\frac{1}{\phi^{*}}-1\right) \phi_{0}$

with $\phi^{*}$ being the volume fraction for the close packing of particles. A represents in eq.(15) the ratio of actual solid volume fraction to the effective volume fraction associated with the solid and the screened fluid.

Buyevich and Kabpsov ${ }^{6}$ considered the flows with strong $(P e \rightarrow 0)$ and negligible $(P e \rightarrow \infty)$ effects of Brownian diffusion in relation to shear effects, to finally formulate the relation valid also for the intermediate $P e$ values. They proposed the following relation for the narrow concentration range that adjoins $\phi^{*}, \phi^{*}-\phi<<\phi, \phi^{*}$, being the closepacked limit for particle volume fraction in suspension $\phi$.

$M(\phi)=-C \ln \ln \frac{1-\phi^{1 / 3}}{\left(\phi^{*}\right)^{1 / 3}}$ 
with $C=z \phi^{*} / 2$, where $z$ is an effective ,coordination number" of a sphere in the close-packed state. Using Eq. (16) together with the empirical formula valid for the low and moderate concentrations

$\mu=\mu_{\text {liquid }}(1-\phi)^{-5 / 2}$ for $\phi \leq \phi^{*}-\phi$

Buyevich and Kabpsov ${ }^{\mathbf{6}}$ developed the expression covering a whole range of concentrations and including both mechanisms: the effect of shear and the effects of Brownian motion. The expression valid at very low shear rates $(P e<<1)$ reads

$$
M_{0}=\frac{1}{\left(1-\phi_{e f f}\right)^{5 / 2}}+1.3\left[\frac{1}{\left(1-\frac{\phi_{e f f}}{\phi^{*}}\right)^{2}}-\sum_{j=0}^{2}(1+j)\left(\frac{\phi_{e f f}}{\phi^{*}}\right)^{j}\right]
$$

and for the flows with high effect of shear $(\mathrm{Pe}>>1)$

$$
M_{\infty}=\frac{1}{\left(1-\phi_{e f f}\right)^{5 / 2}}-C\left[\ln \ln \left(1-\left(\frac{\phi_{\text {eff }}}{\phi^{*}}\right)^{1 / 3}\right)+\sum_{j=1}^{6} \frac{1}{j}\left(\frac{\phi_{\text {eff }}}{\phi^{*}}\right)^{j / 3}\right]
$$

with $C \approx 2$. The relation for the viscosity between $M_{0}$ and $M_{\infty}$ depends on the value of the Peclet number defined as $P e=\frac{\gamma a^{2}}{D_{B}}$

where $a$ is the radius of spherical particles and $D_{\mathrm{B}}$ is the Brownian diffusivity. For any $P e$ one has:

$M=\frac{M_{0}+M_{\infty} P e}{1+P e}$

The model was applied earlier in the context of deagglomeration, modelling by Bałdyga et al. ${ }^{7}$.

In the works by Baxter ${ }^{8}$, Russel ${ }^{9}$ and later Cichocki and Felderhof ${ }^{\mathbf{1 0}}$ the following equation was derived to account for the effects of colloidal forces (cited after Reub and Zukoski $\left.{ }^{11}\right)$ :

$\frac{\mu}{\mu_{0}}=M=1+2.5 \phi_{e f f}+\left(5.9+\frac{1.9}{\tau_{B}}\right) \phi_{e f f}^{2}$

where $\tau_{\mathrm{B}}$ characterizes the strength of attraction. This expression is appropriate for the rather low solid volume fraction values.

When the suspension concentration is so high that fractal aggregates touch each other and overlap, then the overlapped volume fraction can be expressed by $\phi_{e f f}-\phi^{*}$, the number of contacts between primary particles of two overlapping agglomerates of equal size should be proportional to the square of the number of primary particles $\left[\left(\frac{L_{a}}{L_{0}}\right)^{D_{f}}\right]^{2}$ present in this volume, and the number of contact points between the agglomerates of a similar shape (spherical) in a concentrated suspension should be proportional to the number of agglomerates $\frac{\phi_{\text {eff }}}{v_{0}\left(\frac{L_{a}}{L_{0}}\right)^{3}}$. Hence, for the high volume fraction limit the following equation can be used:

$$
M(\phi) \sim\left(\phi_{e f f}-\phi^{*}\right)\left(\frac{L_{a}}{L_{0}}\right)^{2 D_{f}} \frac{\phi_{e f f}}{v_{0}\left(\frac{L_{a}}{L_{0}}\right)^{3}} \cong \frac{C_{1}}{v_{0}} \phi_{e f f}\left(\phi_{e f f}-\phi^{*}\right)\left(\frac{L_{a}}{L_{0}}\right)^{2 D_{f}-3}
$$

The combination of the equations (21) and (23) gives the relation for a wide range of the volume fraction.

\section{DISINTEGRATION OF AGGLOMERATES}

The breakage kernels for agglomerate dispersion are defined by comparing the tensile strength of the agglomerates with the stresses generated in the deagglomeration devices. When the rapture force exceeds the aggregate strength, the break up occurs with a characteristic frequency proportional to the frequency characterizing the phenomenon, which creates stresses, otherwise the rate of breakage is zero. Depending on the process conditions observed in different pieces of equipment, different mechanisms are effective for agglomerate breakage. The hydrodynamic stresses play a significant role in the case of systems with rotating elements and high-pressure devices. For the turbulent flow they can be expressed using the Kolmogorov theory. The frequency of breakup events, $\Gamma$, is then given by:

for $L \leq \lambda_{k}=\frac{v^{\frac{3}{4}}}{\varepsilon^{\frac{1}{4}}} \Gamma=\left\{\begin{array}{c}C_{b}\left(\frac{\varepsilon}{v}\right)^{\frac{1}{2}} \text { if } \sigma_{T}<\mu\left(\frac{\varepsilon}{v}\right)^{\frac{1}{2}} \\ \quad \text { if } \sigma_{T} \geq \mu\left(\frac{\varepsilon}{v}\right)^{\frac{1}{2}}\end{array}\right.$

for $L>\lambda_{k}=\frac{v^{3 / 4}}{\varepsilon^{1 / 4}}: \Gamma=\left\{\begin{array}{c}C_{b} \frac{\varepsilon^{1 / 3}}{L_{i}^{2 / 3}} \text { if } \sigma_{T}<\rho \varepsilon^{2 / 3} L^{2 / 3} \\ 0 \text { if } \sigma_{T} \geq \rho \varepsilon^{2 / 3} L^{2 / 3}\end{array}\right.$

where $C_{\mathrm{b}}$ is a proportionality constant and $\lambda_{K}=\mathrm{v}^{34} / \varepsilon^{1 / 4}$ represents the Kolomogorov microscale. The effect of the suspended particles is taken into account by using the effective viscosity. The rate of energy dissipation, $\varepsilon$, is defined as the average energy dissipation rate in the device, and if necessary, the local values are recalculated from this average one using the information about the distribution of the energy dissipation rate. For the systems with rotating elements the average rate of energy dissipation is calculated from

$\varepsilon=\frac{P}{V \rho}$

where $P$ is the power input calculated from the operating parameters, $V$ is the device volume and $\rho$ is the suspension density.

In the case of high-pressure systems the rate of energy dissipation is calculating from the operating pressure drop, $\Delta p$ :

$\varepsilon=\frac{\Delta p Q}{V \rho}$

where $Q$ is the flow rate. The residence time in any system is calculated from the device volume and the flow rate.

Bead mills represent another type of equipment, where the stresses are generated by the collisions of the milling 
beads. While using the theory of Batchelor and Green12, one can estimate the maximum stresses created during the contacts of beads:

$\tau_{\max }=4.9 \mu_{0} \dot{\gamma}\left[\frac{5}{2}+7.6 \varphi\right]$

where $\varphi$ represents the concentration of the milling beads and $\dot{\gamma}$ is the rate of shear. The breakage kernel in this case is proportional to the number of binary collisions per unit time per unit volume ${ }^{13}$ :

$$
\Gamma= \begin{cases}4 \sqrt{\pi} \beta n_{g}^{2 z}{ }_{b}\left[1-\left(\frac{\varphi}{\varphi_{m}}\right)^{\frac{1}{3}}\right]^{-1} \sqrt{\Theta} & \text { if } \sigma_{T}<\tau_{\max } \\ 0 & \text { if } \sigma_{T} \geq \tau_{\max }\end{cases}
$$

where $n_{b}=\frac{6 \varphi}{\pi d_{b}^{3}}$ is the bead number concentration, $d_{b}$ represents the milling bead diameter, and $\Theta$ is granular temperature. Since not every collision of two particles is effective the equation contains the constant $\beta$ that can be fitted to the experimental data. In ref ${ }^{14}$ is it shown that the collision efficiency strongly depends on the volume fraction of the milling beads. The resulting general relation for the collision frequency reads:

$\Gamma=0.5 \alpha \pi d_{b}^{2} n_{b}^{2} \overline{v_{r}}$

A comparison of eq.(29) with eq.(30) enables to express the average relative velocity between beads, $\bar{v}_{r}$, as a function of granular temperature and bead volume fraction. For the particles with the diameter around $1.8 \mathrm{~mm}$, and the particle number density $n_{\mathrm{b}}=5 \cdot 10^{-6} \mathrm{~m}^{-3}$ for the volume fraction equal to 0.015 , the experimental results show that the collision rate is proportional to $\left(n_{b}^{2}\right)^{0.547}$ so that the collision rate can be expressed as:

$\Gamma=2.6 \cdot 10^{5} d_{b}^{2} n_{b}^{1.15} v_{r}$

which shows that the efficiency of collisions $\beta$ is proportional to $n_{b}^{-0.85}$.

To calculate the granular temperature and the related rate of energy dissipation at micro-scale one can use the model described by Eskin et al. ${ }^{15}$.

The ultrasonic devices are another type of the deagglomeration systems. The main mechanism of generating stresses in ultrasonic devices is based on the cavitation bubble collapse. Cavitation occurs when pressure $p$ falls sufficiently low to form vapour bubbles. When the bubbles are conveyed to the regions of higher pressure, they collapse generating at the same time very high-localized pressure. Surface damage and deagglomeration result from shock waves and microjets generated during such collapse. The semi-empirical relation for the stresses generated during bubble implosion can be estimated from the following relation ${ }^{16}$ :

$\tau_{c}=\alpha \rho c u_{j}$

where $\alpha$ is an empirical constant, $c$ is the velocity of compressional wave in the liquid and $u_{j}$ is the microjet velocity

$u_{j}=\frac{\left(p-p_{v}\right)^{1 / 2}}{0.915 \rho^{1 / 2}}$
In eq.(33) $p_{v}$ is the saturated vapour pressure. The kernel for the frequency of deagglomeration takes the form:

$\Gamma= \begin{cases}C_{b c} v \phi_{e f f} & \text { if } \sigma_{T}<\tau_{c} \\ 0 & \text { if } \sigma_{T} \geq \tau_{c}\end{cases}$

where $v$ represents here the frequency of ultrasonic field.

The mechanisms describing the disintegration of agglomerates are erosion, rapture and shattering. In what follows we consider the erosion and rupture. If the erosion is the dominant breakup mechanism, it occurs on the periphery of the agglomerate. The number of primary aggregates or primary particles (depending on the agglomerate structure, cluster-fractal or fractal) eroded from the agglomerate surface is equal to the number of breakage events that happen during the deagglomeration time:

$N_{b}=\Gamma \cdot \tau$

where $\tau$ is residence time in the deagglomeration device. The relation for the new size of agglomerate based on the conservation of mass considers the fractal structure of agglomerate:

$L_{i, \text { new }}=\left(L_{i, \text { old }}^{D_{f}}-N_{b} L_{a}^{D_{f}}\right)^{1 / D_{f}}$

For the rapture as the main mechanism in the simplest case there are two fragments of agglomerate of the same size per each breakage event:

$L_{i, \text { new }}=\frac{L_{i}}{2^{\frac{N_{b}}{D_{f}}}}$

The examples of the calculations using this simple model are shown in the following figures.

Fig. 2 shows the results of modeling obtained for the system with rotating elements, the Rotor-Stator Mixer, Silverstone type. The analysis of the experimental data ${ }^{7}$ leads to a conclusion that erosion is the mechanism of breakage in the rotor-stator mixer. Hence, this mechanism of deagglomeration is applied in the present work. The experiments and the present calculation were performed using the test rig consisting of an in-line Silverson rotor-stator mixer and the stirred tank arranged in the loop, so that the slurry was kept suspended off the tank base and was flowing through the rotor-stator many times. The experiments were performed by means of varying the rotor speed $(3000-9000 \mathrm{rpm})$ and Aerosil $200 \mathrm{~V}$ was used as a test material. Comparison of model predictions with the experimental data shows that the agreement is good: in the experiments the mean value of the agglomerate diameter for $3000 \mathrm{rpm}$ decreased from $35 \mu \mathrm{m}$ to 15 $\mu \mathrm{m}$, for $5000 \mathrm{rpm}$ further to $10 \mu \mathrm{m}$, for $7000 \mathrm{rpm}$ to $8 \mu \mathrm{m}$ and for $9000 \mathrm{rpm}$ to $5 \mu \mathrm{m}$.

Fig. 3 shows the results of the calculations obtained for the high-pressure system, APV Gaulin 25 Homogenizer. Here, due to the much higher energy input the mechanism of breakage is rapture. For the initial agglomerate size equal to $35 \mu \mathrm{m}$, the experimental data show the variation of the agglomerate size after deagglomeration between $7 \mu \mathrm{m}$ and $1 \mu \mathrm{m}$ for the operating pressure varying between 100 and 500 bar for a different time of the deagglomeration process, which is related to the number of passages through the disintegrating nozzle.

Fig. 4 shows the results for the Stirred Media Mill for erosion as the deagglomeration mechanism. The efficiency 

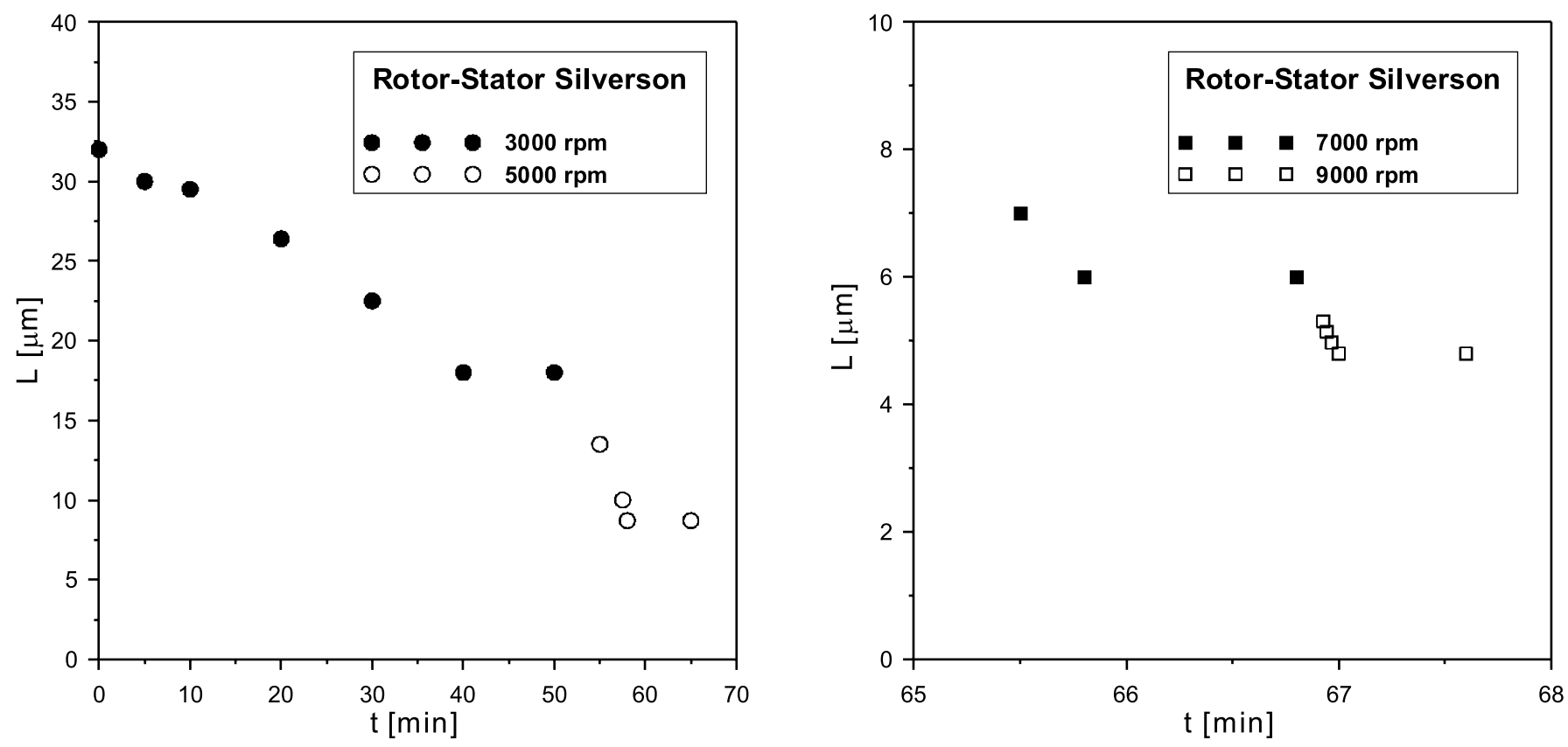

Figure 2. The effect of the rotor speed and deagglomeration time on the agglomerate size; the working volume of the rotorstator mixer, $\mathrm{V}=4.8 \cdot 10^{-5} \mathrm{~m}^{3}$, the flow rate through the system, $\mathrm{Q}=6 \cdot 10^{-4} \mathrm{~m}^{3} / \mathrm{s}$

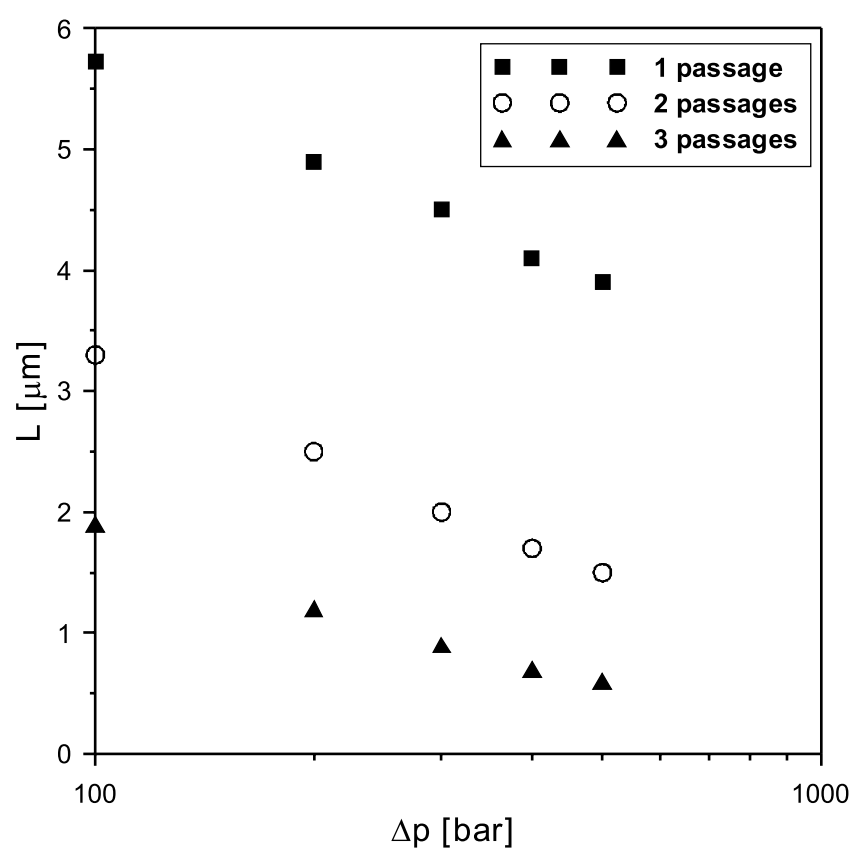

Figure 3. The effect of the operating pressure on the size of agglomerates for a different number of passages through the high-pressure system. APV volume, $\mathrm{V}=4.35 \cdot 10^{-8} \mathrm{~m}^{3}$, flow rate, $\mathrm{Q}=16.5 \cdot 10^{-6} \mathrm{~m}^{3} / \mathrm{s}$

for the frequency of breakage was fitted to the experimental data as mentioned above (see the comment below eq.(31)). The constant $\beta$ for the process parameters: $\varphi=0.3$ and $d_{b}=0.001 \mathrm{~m}$ is equal to about $10^{-8}$. There is a difference in the value of the constant $\beta$ between the one used in ref $^{\mathbf{1 4}}$ and the value given above; this results from the strong influence of the bead milling volume fraction, $\varphi$, that is different in both considered simulations. The granular temperature for the power input $P=2 \cdot 10^{6} \mathrm{~W} / \mathrm{m}^{3}$ was calculated to be $\Theta=0.2481 \mathrm{~m}^{2} / \mathrm{s}^{2}$. For the power input $P=2 \cdot 10^{6} \mathrm{~W} / \mathrm{m}^{3}$ the process time shown in Fig. 4 corresponds to the specific energy value between 10 and $50 \mathrm{MJ} / \mathrm{m}^{3}$. The particle sizes observed in

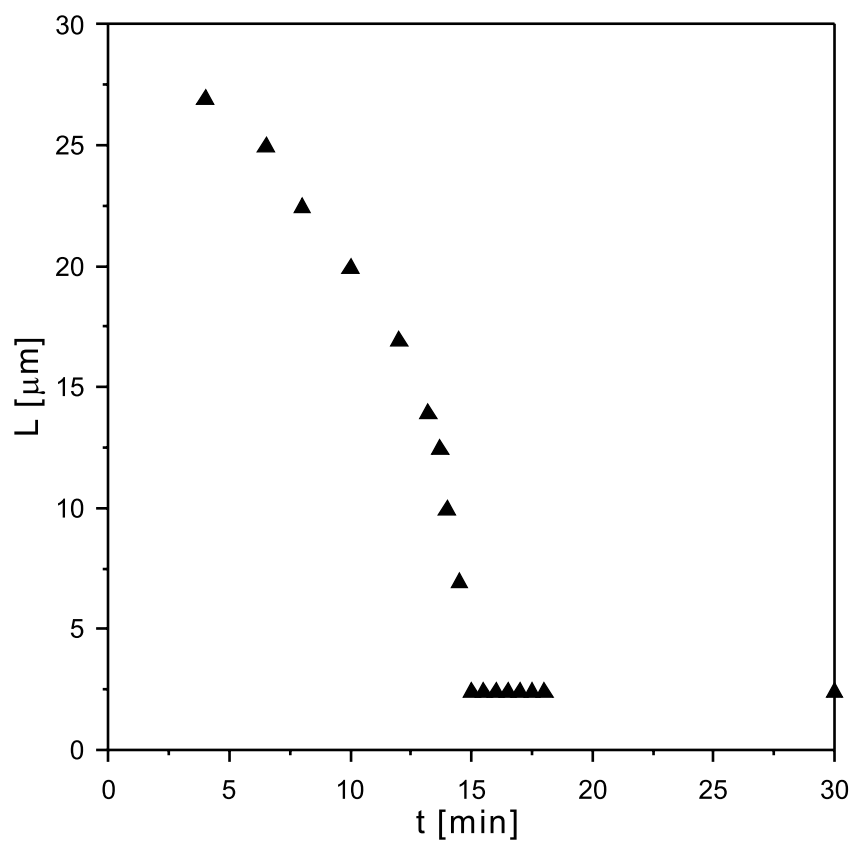

Figure 4. The effect of deaggregation time on the size of aggregate. The collision efficiency $B=10^{-8}$

the simulations for the initial agglomerate size equal to about $35 \mu \mathrm{m}$ agree with the literature experimental data ${ }^{17}$.

The comparison of the effects of energy input on the agglomerate size in different deagglomeration devices is shown in Fig. 5. The high-pressure system and the stirred media mill generate much higher values of specific energy in comparison with the rotor-stator system. Higher values of energy result in smaller size of agglomerates in the product suspension.

\section{CONCLUSIONS}

The effects of the deagglomeration processes result from different mechanisms, depended on many process and 


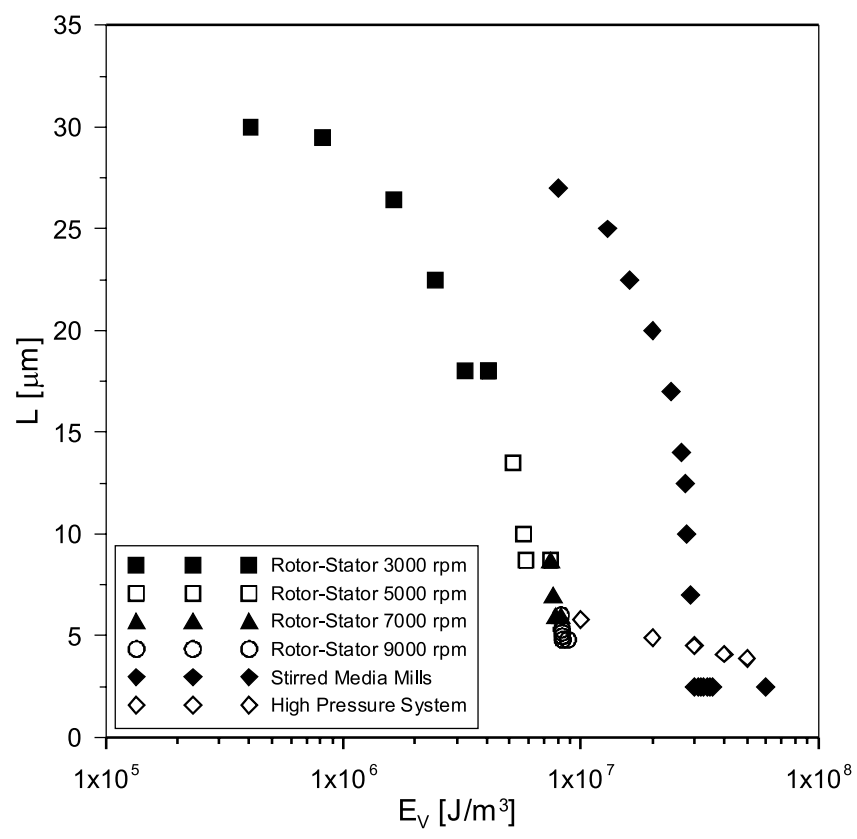

Figure 5. The effect of energy input on the size of the aggregates for different deagglomeration systems

product parameters. However, as shown in this paper, deagglomeration can be described while using relatively simple mechanistic models. The results of the simulation produce good agreement with the experimental data, at least provide a prediction of the trends observed in the experiments. The algorithm of the computations presented in this paper can be very useful in the selection of the equipment for the deagglomeration processes.

\section{ACKNOWLEDGEMENTS}

This study was in part carried out within the project PROFORM (,,Transforming Nano-particles into Sustainable Consumer Products Through Advanced Product and Process Formulation" EC Reference NMP4-CT-2004505645) which was partially funded by the 6th Framework Programme of EC. The content of this paper reflects only the authors' view. The authors gratefully acknowledge the useful discussions held with other partners of the Consortium: BHR Group Limited; Karlsruhe University, Institute of Food Process Engineering; Bayer Technology Services GmbH; University of Loughborough; Unilever UK Central Resources Limited; Birmingham University School of Engineering; Poznan University of Technology, Institute of Chemical Technology and Engineering; Rockfield Software Limited; Centre for Continuum Mechanics, Ljubljana.

\section{NOMENCLATURE}

$c \quad$ - velocity of compressional wave, $\mathrm{m} \mathrm{s}^{-1}$

$D_{\mathrm{f}} \quad$ - fractal dimension of agglomerates

$D_{\mathrm{f} 0} \quad$ - fractal dimension of primary aggregates

$F \quad$ - particle interaction force, $\mathrm{N}$

$H$ - surface-to-surface distance, $\mathrm{m}$

Ha - Hamaker constant, J

$I_{\mathrm{s}} \quad$ - ionic strength, $\mathrm{kmol} \mathrm{m}^{-3}$

$L \quad$ - agglomerate size, $\mathrm{m}$

$L_{\mathrm{a}} \quad$ - primary aggregate size, $\mathrm{m}$

$L_{\mathrm{i}} \quad$ - agglomerate size, $\mathrm{m}$

$L_{0} \quad$ - primary particle size, $\mathrm{m}$ $p \quad$ - pressure, $\mathrm{Pa}$

$R_{0} \quad$ - bubble radius, $\mathrm{m}$

$\mathrm{T} \quad$ - temperature, $\mathrm{K}$

$z \quad$ - valence of ions

$\Gamma \quad$ - breakage kernel, $\mathrm{s}^{-1}$

$\delta_{\mathrm{a}} \quad-$ shape factor for agglomerates

$\delta_{\mathrm{p}} \quad$ - shape factor for aggregates

$\varepsilon \quad-$ rate of energy dissipation per unit mass, $\mathrm{m}^{2} \mathrm{~s}^{-3}$

$\varepsilon_{\mathrm{a}} \quad$ - porosity of agglomerates

$\varepsilon_{0} \quad$ - porosity of primary aggregates

$\theta_{\mathrm{a}} \quad$-packing factor for aggregates in agglomerate

$\theta_{\mathrm{p}} \quad$ - packing factor for primary particles in aggregates

$\lambda_{\mathrm{k}} \quad-$ Kolmogorov microscale, $\mathrm{m}$

$v \quad-$ effective kinematic viscosity, $\mathrm{m}^{2} \mathrm{~s}^{-1}$

$v \quad-$ frequency of ultrasonic field in eq.(34), $\mathrm{s}^{-1}$

$\sigma_{\mathrm{T}} \quad-$ tensile strength of agglomerates, $\mathrm{Pa}$

$\phi_{0} \quad-$ true volume fraction of primary particles

$\phi_{\text {eff }} \quad$ - effective volume fraction of agglomerates

$\psi_{\mathrm{d}} \quad$ - surface potential, V

\section{LITERATURE CITED}

1. Logan, B.E. (1999). Enviromental Transport Processes (pp. 466 - 504). Wiley, New York.

2. Bałdyga, J., Orciuch, W., Makowski, Ł., Malski-Brodzicki, M. \& Malik, K. (2007). Break up of nano-particle clusters in high-shear devices. Chemical Engineering and Processing 46(9), 851 - 861. DOI:10.1016/j.cep.2007.05.016.

3. Tang, S., Ma, Y. \& Shiu, C. (2001). Modeling the mechanical strength of fractal aggregates. Colloid. Surf. A: Phys. Eng. Aspects 180(1 - 2), 7 - 16. DOI:10.1016/S09277757(00)00743-3.

4. Elimelech, M., Gregory, J., Jia, X. \& Williams, R.A. (1995). Particle Deposition and Aggregation. ButterworthHeinemann, Oxford.

5. Collins, J.R. (1996). On the viscosity of concentrated aggregated suspensions. J. Colloid and Interface Sci. 178(1), 361 - 363. DOI:10.1006/jcis.1996.0125.

6. Buyevich, Yu.A. \& Kapbsov, S.K. (1999). Segregation of a fine suspension in channel flow, J. Non-Newt. Fluid. Mech. 86(1 - 2), 157 - 184. PII: S0377-0257(98)00207-9.

7. Bałdyga, J., Orciuch, W., Makowski, L., Malik, K., OzcanTaskin, G., Eagles, W. \& Padron, G. (2008). Dispersion of nanoparticle clusters in a rotor-stator mixer. Industrial and Engineering Chemistry Research 47(10), 3652 - 3663. DOI: 10.1021/ie070899u.

8. Baxter, R.J. \& Percus-Yevick (1968). Equation for Hard Spheres with Surface Adhesion. Journal of Chemical Physics 49, 2770 - 2774. DOI: 10.1063/1.1670482.

9. Russel, W.B. (1984). The Huggins coefficient as a means for characterizing suspended particles. J. Chem. Soc. Faraday Trans. 2, 80, 31 - 41. DOI: 10.1039/F29848000031.

10. Cichocki, B. \& Felderhof, B.U. (1990). Diffusion coefficients and effective viscosity of suspensions of sticky hard spheres with hydrodynamic interactions. J. Chem. Phys. 93(6), 4427 - 4432. DOI:10.1063/1.459688.

11. Rueb, C.J. \& Zukoski, C.F. (1998). Rheology of suspensions of weakly attractive particles: approach to gelation. J. Rheol. 42(6), 1451 - 1476. DOI: 10.1122/1.550966.

12. Batchelor, G.K. \& Green, J.T. (1972). The hydrodynamic interaction of two small freely-moving spheres in a linear flow field. J. Fluid Mech. 56(2), 375 - 400. DOI:10.1017/ S0022112072002927.

13. Gidaspow, D. (1994). Multiphase Flow and Fluidization: Continuum and Kinetic Theory Descriptions. Academic Press, Boston.

14. Changfu You, Hailiang Zhao, Yi Cai, Haiying Qi \& Xuchang $\mathrm{Xu}$ (2004). Experimental investigation of 
interparticle collision rate in particulate flow. Multiphase Flow 30(9), 1121 - 1138. DOI:10.1016/j.ijmultiphaseflow. 2004.05.009.

15. Eskin, D., Zhupanska, O., Hamey, R., Moudgil, B. \& Scarlett, B. (2005). Microhydrodynamics of stirred media milling. Powder Technology 156,(2 - 3), 95 - 102. DOI:10.1016/ j.powtec.2005.04.004.

16. Crum, L. (1998). Cavitation microjets as a contributory mechanisms for renal disintegration in ESWL. J. Urol. 140(6), 1587 - 1590. PMID: 3057239.

17. Stender H. -H, Kwade, A. \& Schwedes, J. (2004). Stress energy distribution in different stirred media mill geometries. Int. J. Miner. Process 74S(1), S103 - S117. DOI:10.1016/j.minpro.2004.07.003. 